\title{
UNIFORM NUMERICAL APPROXIMATION FOR PARAMETER DEPENDENT SINGULARLY PERTURBED PROBLEM WITH INTEGRAL BOUNDARY CONDITION
}

\author{
MUSTAFA KUDU, ILHAME AMIRALI, AND GABIL M. AMIRALIYEV
}

Received 22 November, 2017

\begin{abstract}
In this paper, a parameter-uniform numerical method for a parameterized singularly perturbed ordinary differential equation containing integral boundary condition is studied. Asymptotic estimates on the solution and its derivatives are derived. A numerical algorithm based on upwind finite difference operator and an appropriate piecewise uniform mesh is constructed. Parameter-uniform error estimate for the numerical solution is established. Numerical results are presented, which illustrate the theoretical results.
\end{abstract}

2010 Mathematics Subject Classification: 65L11; 65L12; 65L20; 65L70

Keywords: parameterized problem, singular perturbation, uniform convergence, finite difference scheme, Shiskin mesh, integral boundary condition

\section{INTRODUCTION}

In this paper, we consider the following parameterized singular perturbation problem with integral boundary condition arising in many scientific applications [16, 23](see also references therein):

$$
\begin{gathered}
\varepsilon u^{\prime}+f(t, u, \lambda)=0, t \in \Omega=(0, T], T>0, \\
u(0)+\int_{0}^{T} c(s) u(s) d s=A, \\
u(T)=B,
\end{gathered}
$$

where $\varepsilon \in(0,1]$ is the perturbation parameter, $\lambda$ is known as the control parameter, $A$ and $B$ are given constants. The functions $c(t) \geq 0$ and $f(t, u, \lambda)$ are assumed to be sufficiently continuously differentiable for our purpose in $\bar{\Omega}=\Omega \cup\{t=0\}$ and $\Omega \times \mathbb{R}^{2}$ respectively and moreover

$$
0<\alpha \leq \frac{\partial f}{\partial u} \leq a^{*}<\infty
$$




$$
0<m_{1} \leq\left|\frac{\partial f}{\partial \lambda}\right| \leq M_{1}<\infty .
$$

By a solution of (1.1)-(1.3) we mean $\{u(t), \lambda\} \in C^{1}[0, T] \times \mathbb{R}$ for which problem (1.1)-(1.3) is satisfied.

Singularly perturbed differential equations are typically characterized by a small parameter $\varepsilon$ multiplying some or all of the highest order terms in the differential equation as normally boundary layers occur in their solutions. These equations play an important role in today's advanced scientific computations. Many mathematical models starting from fluid dynamics to the problems in mathematical biology are modelled by singularly perturbed problems. Typical examples include high Reynold's number flow in the fluid dynamics, heat transport problem etc. For more details on singular perturbation, one can refer to the books $[10,12,19,21]$ and the references therein. The numerical analysis of singular perturbation cases has always been far from trivial because of the boundary layer behavior of the solution. Such problem undergo rapid changes within very thin layers near the boundary or inside the problem domain [19,21]. It is well known that standard numerical methods for solving such problems are unstable and fail to give accurate results when the perturbation parameter is small. Therefore, it is important to develop suitable numerical methods to these problems, whose accuracy does not depend on the parameter value, i.e. methods that are convergence $\varepsilon$-uniformly. For the various approaches on the numerical solution of differential equations with steep gradients and continuous solutions we may refer to the studies $[8,10-12]$. Parameterized boundary value problems have been considered by many researchers for many years. Such problems arise in physical chemistry and physics, describing the exothermic and isothermal chemical reactions, the steady-state temperature distributions, the oscillation of a mass attached by two springs lead to a differential equation with a parameter [18, 22]. An overview of some existence and uniqueness results and applications of parameterized equations may be obtained, for example, in $[13,16,18,22]$ (see, also references therein). In $[18,22]$, the authors have also been considered some approximating aspects of this kind of problems. But in the above-mentioned papers, algorithms are only concerned with the regular cases (i.e., when the boundary layers are absent). In recent years, many researchers presented the numerical methods for the singular perturbation cases of parameterized problems. Uniform convergent finite-difference schemes for solving parameterized singularly perturbed two-point boundary value problems have been considered in [2, 3, 9, 17, 24, 25](see, also references therein). In [2,3,17] authors used boundary layer technique for solving analogous problem. A methodology based on the homotopy analysis technique to approximate the analytic solution was investigated in [24,25]. Also it is well known that nonlinear differential equations with integral boundary conditions have been used in description of many phenomena in the applied sciences, e.g., heat conduction, chemical engineering, underground water flow and so on $[6,15,20]$. Therefore, boundary value problems involving integral 
boundary conditions have been studied by many authors $[1,4,5,7,11,14,16,23]$ (see, also references therein). Some approximating aspects of this kind of problems in the regular cases, i.e, in absence of layers, were investigated in $[4,11,14,16,23]$. In recent years, many researchers considered the singularly perturbed case for these problems. In $[1,5,7]$ authors develop a finite difference scheme on Shishkin mesh for problem with integral boundary conditions and proved that the method is nearly first order convergent except for a logarithmic factor. A hybrid scheme, which is second order convergent on Shishkin mesh was discussed in [7]. For the numerical methods, concerning to second order singularly perturbed differential equations with integral boundary conditions can be seen e.g., [5]. In this paper, as far as we know the numerical solution of the singularly perturbed boundary value problem containing both control parameter and integral condition is first being considered. For the numerical solution of such problems, requires specific approach in constructing of the appropriate difference scheme and examining the error analysis. The scheme is constructed by the method of integral identities with the use of appropriate quadrature rules with the remainder terms in integral form. We show that the proposed scheme is uniformly convergent in the discrete maximum norm accuracy of $O\left(N^{-1} \ln N\right)$ on Shishkin meshes. First, the asymptotic estimates for the continuous solution are given in Section 2, which are needed in later sections for the analysis of appropriate numerical solution. In Section 3, we describe the finite discretization and give the difference scheme on a piecewise uniform grid. In Section 4, the convergence analysis is carried out. Finally, in Section 5 presents some numerical results to confirm the theoretical analysis. Henceforth, $C$ and $c$ denote the generic positive constants independent of both the perturbation parameter $\varepsilon$ and mesh parameter $N$. Such subscripted constants are also independent of $\varepsilon$ and mesh parameter, but whose values are fixed.

\section{ASYMPTOTIC BEHAVIOR OF THE EXACT SOLUTION}

In this section, we give a priori estimates for the solution and its derivatives of the problem (1.1)-(1.3), which indicate the asymptotic behavior of the solution and its first derivative in respect to perturbation parameter. These estimates are unimprovable in terms of the view of behavior in $\varepsilon$ and will be used in order to analyse the numerical solution. We also denote $\|g\|_{\infty}=\max _{[0, T]}|g(t)|$ for any $g \in C[0, T]$.

Lemma 2.1 The solution $\{u(t), \lambda\}$ of the problem (1.1)-(1.3) satisfies the following bounds:

$$
\begin{gathered}
|\lambda| \leq c_{0}, \\
\|u\|_{\infty} \leq c_{1},
\end{gathered}
$$

where

$$
c_{0}=m_{1}^{-1}\left\{\frac{\alpha|A|}{e^{\alpha T}-1}+\frac{|B| a^{*}\left(1-\|c\|_{\infty} T\right)}{m_{1}\left(e^{a^{*} T}-1\right)}+\|F\|_{\infty}\right\},
$$




$$
\begin{gathered}
c_{1}=|u(0)|+\alpha^{-1}\left(\|F\|_{\infty}+|\lambda| M_{1}\right)=|u(0)|+\alpha^{-1}\left(\|F\|_{\infty}+c_{0} M_{1}\right), \\
\left|u^{\prime}(t)\right| \leq C\left(1+\frac{1}{\varepsilon} e^{-\frac{\alpha t}{\varepsilon}}\right), t \in[0, T],
\end{gathered}
$$

provided $a \in C^{1}[0, T]$ and $\left|\frac{\partial f}{\partial t}\right| \leq C$ for $t \in[0, T]$ and $|u| \leq c_{1},|\lambda| \leq c_{0}$.

Proof. The quasilinear equation (1.1) can be written as

$$
\varepsilon u^{\prime}+a(t) u=F(t)+\lambda b(t), \quad t \in[0, T],
$$

where

$$
\begin{gathered}
a(t)=\frac{\partial f}{\partial u}(t, \tilde{u}, \tilde{\lambda}), \\
b(t)=-\frac{\partial f}{\partial \lambda}(t, \tilde{u}, \tilde{\lambda}),
\end{gathered}
$$

$\widetilde{u}=\gamma u, \widetilde{\lambda}=\gamma \lambda(0<\gamma<1)$-intermediate values.

Integrating (2.4), (1.3) we have

$$
u(t)=B e^{\frac{1}{\varepsilon} \int_{t}^{T} a(\xi) d \xi}-\frac{1}{\varepsilon} \int_{t}^{T} F(\xi) e^{\frac{1}{\varepsilon} \int_{t}^{\xi} a(\eta) d \eta} d \xi+\frac{\lambda}{\varepsilon} \int_{t}^{T} b(\xi) e^{\frac{1}{\varepsilon} \int_{t}^{\xi} a(\eta) d \eta} d \xi
$$

from which, after using the integral boundary condition (1.2), it follows that,

$$
\begin{gathered}
B e^{\frac{1}{\varepsilon} \int_{0}^{T} a(\xi) d \xi}-\frac{1}{\varepsilon} \int_{0}^{T} F(\xi) e^{\frac{1}{\varepsilon} \int_{0}^{\xi} a(\eta) d \eta} d \xi+\frac{\lambda}{\varepsilon} \int_{0}^{T} b(\xi) e^{\frac{1}{\varepsilon} \int_{0}^{\xi} a(\eta) d \eta} d \xi \\
+B \int_{0}^{T} c(s) e^{\frac{1}{\varepsilon} \int_{s}^{T} a(\xi) d \xi} d s-\frac{1}{\varepsilon} \int_{0}^{T} c(s)\left[\int_{s}^{T} F(\xi) e^{\frac{1}{\varepsilon} \int_{s}^{\xi} a(\eta) d \eta} d \xi\right] d s \\
+\frac{\lambda}{\varepsilon} \int_{0}^{T} c(s)\left[\int_{s}^{T} b(\xi) e^{\frac{1}{\varepsilon} \int_{s}^{\xi} a(\eta) d \eta} d \xi\right] d s=A
\end{gathered}
$$

and

$$
\begin{gathered}
\lambda=\frac{A}{\frac{1}{\varepsilon} \int_{0}^{T} b(\xi) e^{\frac{1}{\varepsilon} \int_{0}^{\xi} a(\eta) d \eta} d \xi+\frac{1}{\varepsilon} \int_{0}^{T} b(\xi)\left[\int_{s}^{T} c(s) e^{\frac{1}{\varepsilon} \int_{s}^{\xi} a(\eta) d \eta} d s\right] d \xi} \\
-\frac{B\left(e^{\frac{1}{\varepsilon} \int_{0}^{T} a(\xi) d \xi}+\int_{0}^{T} c(s) e^{\frac{1}{\varepsilon} \int_{s}^{T} a(\xi) d \xi} d s\right)}{\frac{1}{\varepsilon} \int_{0}^{T} b(\xi) e^{\frac{1}{\varepsilon} \int_{0}^{\xi} a(\eta) d \eta} d \xi+\frac{1}{\varepsilon} \int_{0}^{T} b(\xi)\left[\int_{s}^{T} c(s) e^{\frac{1}{\varepsilon} \int_{s}^{\xi} a(\eta) d \eta} d s\right] d \xi} \\
+\frac{\frac{1}{\varepsilon} \int_{0}^{T} F(\xi) e^{\frac{1}{\varepsilon} \int_{0}^{\xi} a(\eta) d \eta} d \xi+\frac{1}{\varepsilon} \int_{0}^{T} F(\xi)\left[\int_{s}^{T} c(s) e^{\frac{1}{\varepsilon} \int_{s}^{\xi} a(\eta) d \eta} d s\right] d \xi}{\frac{1}{\varepsilon} \int_{0}^{T} b(\xi) e^{\frac{1}{\varepsilon} \int_{0}^{\xi} a(\eta) d \eta} d \xi+\frac{1}{\varepsilon} \int_{0}^{T} b(\xi)\left[\int_{s}^{T} c(s) e^{\frac{1}{\varepsilon} \int_{s}^{\xi} a(\eta) d \eta} d s\right] d \xi} .
\end{gathered}
$$


As $c(t) \geq 0$, then after applying the mean value theorem for integrals, we deduce that,

$$
\left|\frac{\frac{1}{\varepsilon} \int_{0}^{T} F(\xi) e^{\frac{1}{\varepsilon} \int_{0}^{\xi} a(\eta) d \eta} d \xi+\frac{1}{\varepsilon} \int_{0}^{T} F(\xi)\left[\int_{s}^{T} c(s) e^{\frac{1}{\varepsilon} \int_{s}^{\xi} a(\eta) d \eta} d s\right] d \xi}{\frac{1}{\varepsilon} \int_{0}^{T} b(\xi) e^{\frac{1}{\varepsilon} \int_{0}^{\xi} a(\eta) d \eta} d \xi+\frac{1}{\varepsilon} \int_{0}^{T} b(\xi)\left[\int_{s}^{T} c(s) e^{\frac{1}{\varepsilon} \int_{s}^{\xi} a(\eta) d \eta} d s\right] d \xi}\right| \leq m_{1}^{-1}\|F\|_{\infty}
$$

and

$$
\begin{gathered}
\left|\frac{B\left(e^{\frac{1}{\varepsilon} \int_{0}^{T} a(\xi) d \xi}+\int_{0}^{T} c(s) e^{\frac{1}{\varepsilon} \int_{s}^{T} a(\xi) d \xi} d s\right)}{\frac{1}{\varepsilon} \int_{0}^{T} b(\xi) e^{\frac{1}{\varepsilon} \int_{0}^{\xi} a(\eta) d \eta} d \xi+\frac{1}{\varepsilon} \int_{0}^{T} b(\xi)\left[\int_{s}^{T} c(s) e^{\frac{1}{\varepsilon} \int_{s}^{\xi} a(\eta) d \eta} d s\right] d \xi}\right| \\
\leq \frac{|B|\left(1+\|c\|_{\infty} T\right)}{m_{1} \varepsilon^{-1} \int_{0}^{T} e^{\frac{1}{\varepsilon} \int_{\xi}^{T} a(\eta) d \eta} d \xi} \\
\leq \frac{|B|\left(1+\|c\|_{\infty} T\right)}{m_{1}(a *)^{-1}\left(1-e^{-\frac{a^{*} T}{\varepsilon}}\right)} \leq \frac{|B|\left(1+\|c\|_{\infty} T\right)}{m_{1}\left(a^{*}\right)^{-1}\left(1-e^{-a^{*} T}\right)},(\varepsilon \leq 1) .
\end{gathered}
$$

Also, for the first term in right side of (2.5) for $\varepsilon \leq 1$ values, we get

$$
\begin{gathered}
\left|\frac{A}{\frac{1}{\varepsilon} \int_{0}^{T} b(\xi) e^{\frac{1}{\varepsilon} \int_{0}^{\xi} a(\eta) d \eta} d \xi+\frac{1}{\varepsilon} \int_{0}^{T} b(\xi)\left[\int_{s}^{T} c(s) e^{\frac{1}{\varepsilon} \int_{s}^{\xi} a(\eta) d \eta} d s\right] d \xi}\right| \\
\leq \frac{|A|}{\frac{1}{\varepsilon} \int_{0}^{T} b(\xi) e^{\frac{1}{\varepsilon} \int_{0}^{\xi} a(\eta) d \eta} d \xi} \\
\leq \frac{|A|}{m_{1} \alpha^{-1}\left(e^{\frac{\alpha T}{\varepsilon}}-1\right)} \leq \frac{|A|}{m_{1} \alpha^{-1}\left(e^{\alpha T}-1\right)} \leq \frac{\alpha|A|}{m_{1}\left(e^{\alpha T}-1\right)} .
\end{gathered}
$$

The relation (2.5), by taking into consideration here (2.6)-(2.8), immediately leads to (2.1).

Now, integrating (2.4), we have

$$
u(t)=u(0) e^{-\frac{1}{\varepsilon} \int_{0}^{t} a(\eta) d \eta}+\frac{1}{\varepsilon} \int_{0}^{t} \Phi(\xi) e^{-\frac{1}{\varepsilon} \int_{\xi}^{t} a(\eta) d \eta} d \xi ; \Phi(s)=F(s)-\lambda b(s),
$$

from which, by setting the integral boundary condition (1.2), we get

$$
u(0)=\frac{A-\frac{1}{\varepsilon} \int_{0}^{T} c(s)\left[\int_{0}^{s} \Phi(\xi) e^{-\frac{1}{\varepsilon} \int_{\xi}^{s} a(\eta) d \eta} d \xi\right] d s}{1+\int_{0}^{T} c(s) e^{-\frac{1}{\varepsilon} \int_{0}^{s} a(\xi) d \xi} d s} .
$$


Since $c(t)$ is nonnegative, then

$$
\begin{aligned}
& |u(0)|=\left|\frac{A-\frac{1}{\varepsilon} \int_{0}^{T} c(s)\left[\int_{0}^{s} \Phi(\xi) e^{-\frac{1}{\varepsilon} \int_{\xi}^{s} a(\eta) d \eta} d \xi\right] d s}{1+\int_{0}^{T} c(s) e^{-\frac{1}{\varepsilon} \int_{0}^{s} a(\xi) d \xi} d s}\right| \\
& \leq|A|+\frac{1}{\varepsilon} \int_{0}^{T} c(s)\left[\int_{0}^{s}|\Phi(\xi)| e^{-\frac{1}{\varepsilon} \int_{\xi}^{s} a(\eta) d \eta} d \xi\right] d s \\
& \leq|A|+\frac{1}{\varepsilon}\|c\|_{\infty}\left(\|F\|_{\infty}+M_{1} c_{0}\right) \alpha^{-1} \varepsilon \int_{0}^{T}\left(1-e^{-\frac{\alpha s}{\varepsilon}}\right) d s \\
& \leq|A|+\alpha^{-1}\|c\|_{\infty} T\left(\|F\|_{\infty}+M_{1} c_{0}\right) .
\end{aligned}
$$

Next, by virtue of maximum principle we have

$$
\begin{aligned}
& \|u\|_{\infty} \leq|u(0)|+\alpha^{-1}\|F-b \lambda\|_{\infty} \\
& \leq|u(0)|+\alpha^{-1}\left(\|F\|_{\infty}+|\lambda| M_{1}\right),
\end{aligned}
$$

which, after taking into account (2.1) and (2.9) leads to (2.2).

To prove (2.3), first we estimate $u^{\prime}(0)$ :

$$
\left|u^{\prime}(0)\right| \leq \frac{\mid F(0)-a(0) u(0)-b(0) \lambda) \mid}{\varepsilon} \leq \frac{C}{\varepsilon} .
$$

Differentiating, now the equation (2.4), we have

$$
\varepsilon v^{\prime \prime}+p(t) v^{\prime}=g(t),
$$

with

So

$$
v=u^{\prime}, p(t)=\frac{\partial f}{\partial u}(t, u(t), \lambda) \text { and } g(t)=\frac{\partial f}{\partial t}(t, u(t), \lambda) .
$$

$$
v(t)=v(0) e^{-\frac{1}{\varepsilon} \int_{0}^{t} a(s) d s}+\frac{1}{\varepsilon} \int_{0}^{t} g(s) e^{-\frac{1}{\varepsilon} \int_{s}^{t} a(\xi) d \xi} d s .
$$

Since $p(t) \geq \alpha>0$ and $|g(t)| \leq C$, for $v(t)$ we then obtain

$$
\begin{aligned}
|v(t)| & \leq \frac{C}{\varepsilon} e^{\frac{-\alpha t}{\varepsilon}}+\frac{C}{\varepsilon} \int_{0}^{t} e^{\frac{-\alpha(t-s)}{\varepsilon}} d s \\
& \leq \frac{C}{\varepsilon} e^{\frac{-\alpha t}{\varepsilon}}+C\left(1-e^{\frac{-\alpha t}{\varepsilon}}\right)
\end{aligned}
$$

which implies validity of (2.3). 


\section{DISCRETE PROBLEM}

Let $\omega_{N}$ be any non-uniform mesh on $\Omega$ :

$$
\omega_{N}=\left\{0<t_{1}<t_{2}<\ldots<t_{N-1}<t_{N}=T\right\}
$$

and $\bar{\omega}_{N}=\omega_{N} \cup\{t=0\}$. For each $i \geq 1$, we set the step size $h_{i}=t_{i}-t_{i-1}$. To simplify the notation we set $g_{i}=g\left(t_{i}\right)$ for any function $g(t)$, while $g_{i}^{N}$ denotes an approximation of $g(t)$ at $t_{i}$.

For any mesh function $\left\{w_{i}\right\}$ defined on $\bar{\omega}_{N}$ we use

$$
\begin{aligned}
w_{\bar{t}, i} & =\left(w_{i}-w_{i-1}\right) / h_{i}, \\
\|w\|_{\infty} & \equiv\|w\|_{\infty, \bar{\omega}_{N}}:=\max _{0 \leq i \leq N}\left|w_{i}\right| .
\end{aligned}
$$

To obtain approximation for (1.1) we integrate (1.1) over $\left(t_{i-1}, t_{i}\right)$ :

$$
\varepsilon u_{\bar{t}, i}+h_{i}^{-1} \int_{t_{i-1}}^{t_{i}} f(t, u(t), \lambda) d t=0,1 \leq i \leq N,
$$

which yields the relation

$$
\varepsilon u_{\bar{t}, i}+f\left(t_{i}, u_{i}, \lambda\right)+R_{i}=0,1 \leq i \leq N,
$$

with local truncation error

$$
R_{i}=-h_{i}^{-1} \int_{t_{i-1}}^{t_{i}}\left(t-t_{i-1}\right) \frac{d}{d t} f(t, u(t), \lambda) d t .
$$

To define an approximation for the boundary condition (1.2), here we use the composite right-side rectangle rule:

$$
u(0)+\int_{0}^{T} c(s) u(s) d s=u_{0}+\sum_{i=1}^{N} h_{i} c_{i} u_{i}+r
$$

with remainder term

$$
r=-\sum_{i=1}^{N} \int_{t_{i-1}}^{t_{i}}\left(t-t_{i-1}\right) \frac{d}{d t}(c(t) u(t)) d t .
$$

Consequently

$$
u_{0}+\sum_{i=1}^{N} h_{i} c_{i} u_{i}+r=A .
$$

Neglecting $R_{i}$ and $r$ in (3.1) and (3.4), we propose the following difference scheme for approximating (1.1)-(1.3):

$$
\varepsilon u_{\bar{t}, i}^{N}+f\left(t_{i}, u_{i}^{N}, \lambda^{N}\right)=0,1 \leq i \leq N,
$$




$$
\begin{gathered}
u_{0}^{N}+\sum_{i=1}^{N} h_{i} c_{i} u_{i}^{N}=A, \\
u_{N}^{N}=B .
\end{gathered}
$$

The difference scheme (3.5)-(3.7), in order to be $\varepsilon$-uniform convergent, we will use the Shishkin mesh. For an even number $N$, the piecewise uniform mesh takes $N / 2$ points in the interval $[0, \sigma]$ and also $N / 2$ points in the interval $[\sigma, T]$, where the transition point $\sigma$, which separates the fine and coarse portions of the mesh, is obtained by taking

$$
\sigma=\min \left\{\frac{T}{2}, \alpha^{-1} \varepsilon \ln \varepsilon\right\} .
$$

In practice one usually has $\sigma \leq T$, so the mesh is fine on $[0, \sigma]$ and coarse on $[\sigma, T]$. Hence, if we denote by $h^{(1)}$ and $h^{(2)}$ the step size in $[0, \sigma]$ and $[\sigma, T]$, respectively, we have

$$
\begin{gathered}
h^{(1)}=2 \sigma N^{-1}, \quad h^{(2)}=2(T-\sigma) N^{-1}, \\
h^{(1)} \leq T N^{-1}, T N^{-1} \leq h^{(2)}<2 T N^{-1}, \quad h^{(1)}+h^{(2)}=2 T N^{-1},
\end{gathered}
$$

so

$$
\bar{\omega}_{N}=\left\{\begin{array}{cc}
t_{i}=i h^{(1)}, & \text { for } i=0,1, \ldots, N / 2 ; h^{(1)}=2 \sigma / N, \\
t_{i}=\sigma+(i-N / 2) h^{(2)}, & \text { for } i=N / 2+1, \ldots, N ; h^{(2)}=2(T-\sigma) / N .
\end{array}\right.
$$

In the rest of the paper we only consider this mesh.

\section{UNIFORM ERROR ESTIMATES}

To investigate the convergence of the method, note that the error functions $z_{i}^{N}=$ $u_{i}^{N}-u_{i}, 0 \leq i \leq N, \mu^{N}=\lambda^{N}-\lambda$ are the solution of the discrete problem

$$
\begin{gathered}
\varepsilon z_{\bar{t}, i}^{N}+f\left(t_{i}, u_{i}^{N}, \lambda^{N}\right)-f\left(t_{i}, u_{i}, \lambda\right)=R_{i}, 1 \leq i \leq N, \\
z_{0}^{N}+\sum_{i=1}^{N} h_{i} c_{i} z_{i}^{N}-r=0, \\
z_{N}^{N}=0 .
\end{gathered}
$$

where the truncation errors $R_{i}$ and $r$ are given by (3.2) and (3.3), respectively.

Lemma 4.1. The solution of the first order difference equation

$$
y_{i}=q_{i} y_{i-1}+\varphi_{i}, \quad 1 \leq i \leq N
$$

can be expressed in the following forms:

$$
y_{i}=y_{0} Q_{i}+\sum_{k=1}^{i} \varphi_{k} Q_{i-k}
$$


or

$$
y_{i}=y_{N} Q_{N-i}^{-1}-\sum_{k=i+1}^{N} \varphi_{k} Q_{k-i}^{-1}
$$

where

$$
Q_{i-k}=\left\{\begin{array}{cc}
1, & k=i, \\
\prod_{\ell=k+1}^{i} q_{\ell}, & 1 \leq k \leq i-1 .
\end{array}\right.
$$

The relations (4.4) and (4.5) can be easily verified by induction in $i$.

Lemma 4.2. Under the above assumptions of Section 1 and Lemma 2.1, for the error functions $R$ and $r$, the following estimates hold:

$$
\begin{gathered}
\|R\|_{\infty, \omega_{N}} \leq C N^{-1} \ln N, \\
|r| \leq C N^{-1} \ln N .
\end{gathered}
$$

Proof. From explicit expression (3.2) for $R_{i}$, on an arbitrary mesh we have

$$
\begin{gathered}
\left|R_{i}\right| \leq h_{i}^{-1} \int_{t_{i-1}}^{t_{i}}\left(t-t_{i-1}\right)\left|\frac{\partial f}{\partial t}(t, u(t), \lambda)+\frac{\partial f}{\partial u}(t, u(t), \lambda) u^{\prime}(t)\right| d t \\
\leq C h_{i}^{-1} \int_{t_{i-1}}^{t_{i}}\left(t-t_{i-1}\right)\left(1+\left|u^{\prime}(t)\right|\right) d t, 1 \leq i \leq N .
\end{gathered}
$$

This inequality together with (2.3) enables us to write

$$
\left|R_{i}\right| \leq C\left\{h_{i}^{-1}+h_{i}^{-1} \varepsilon^{-1} \int_{t_{i-1}}^{t_{i}}\left(t-t_{i-1}\right) e^{-\alpha t / \varepsilon} d t\right\}, 1 \leq i \leq N
$$

in which

$$
h_{i}= \begin{cases}h^{(1)}, & 1 \leq i \leq N / 2, \\ h^{(2)}, & N / 2+1 \leq i \leq N .\end{cases}
$$

We consider first the case $\sigma=T / 2$ and so $T / 2 \leq \alpha^{-1} \varepsilon \ln N$ and $h^{(1)}=h^{(2)}=$ $T N^{-1}$. Hereby, since

$$
h_{i}^{-1} \varepsilon^{-1} \int_{t_{i-1}}^{t_{i}}\left(t-t_{i-1}\right) e^{-\alpha t / \varepsilon} d t \leq \varepsilon^{-1} h^{(1)} \leq \frac{2 \ln N}{\alpha T} \frac{T}{N}=2 \alpha^{-1} N^{-1} \ln N,
$$

it follows from (4.8) that

$$
\left|R_{i}\right| \leq C N^{-1} \ln N, 1 \leq i \leq N .
$$

We now consider the case $\sigma=\alpha^{-1} \varepsilon \ln N$ and estimate $R_{i}$ on $[0, \sigma]$ and $[\sigma, T]$ separately. In the layer region $[0, \sigma]$, inequality $(4.8)$ reduces to

$$
\left|R_{i}\right| \leq C\left(1+\varepsilon^{-1}\right) h^{(1)}=C\left(1+\varepsilon^{-1}\right) \frac{\alpha^{-1} \varepsilon \ln N}{N / 2}, 1 \leq i \leq N / 2 .
$$


Hence

$$
\left|R_{i}\right| \leq C N^{-1} \ln N, 1 \leq i \leq N / 2 .
$$

It remains to estimate $R_{i}$ for $N / 2+1 \leq i \leq N$. In this case we are able to write (4.8) as

$$
\left|R_{i}\right| \leq C\left\{h^{(2)}+\alpha^{-1}\left(e^{-\frac{\alpha t_{i-1}}{\varepsilon}}-e^{-\frac{\alpha t_{i}}{\varepsilon}}\right)\right\}, N / 2+1 \leq i \leq N .
$$

Since $t_{i}=\alpha^{-1} \varepsilon \ln N+(i-N / 2) h^{(2)}$ it follows that:

$$
e^{-\frac{\alpha t_{i-1}}{\varepsilon}}-e^{-\frac{\alpha t_{i}}{\varepsilon}}=\frac{1}{N} e^{-\frac{\alpha\left(i-1-\frac{N}{2}\right) h^{(2)}}{\varepsilon}}\left(1-e^{-\frac{\alpha h^{(2)}}{\varepsilon}}\right)<N^{-1}
$$

and this together with (4.11) to give the bound

$$
\left|R_{i}\right| \leq C N^{-1} .
$$

The inequalities (4.9), (4.10) and (4.12) finish the proof of (4.6).

Finally, we estimate the remainder term $r$. From the explicit expressiom (3.3) we obtain

$$
|r| \leq \sum_{i=1}^{N} \int_{t_{i-1}}^{t_{i}} c(t)\left|\left(t-t_{i-1}\right)\right|\left|u^{\prime}(t)\right| d t, \quad 1 \leq i \leq N,
$$

This inequality together with (2.3) enable use to write

$$
|r| \leq\|c\|_{\infty} C \sum_{i=1}^{N} h_{i} \int_{t_{i-1}}^{t_{i}}\left(1+\frac{1}{\varepsilon} e^{-\frac{\alpha t}{\varepsilon}}\right) d t, 1 \leq i \leq N .
$$

From (4.13), the validity of (4.7) follows:

$$
\begin{gathered}
|r| \leq C \sum_{i=1}^{N / 2} h^{(1)} \int_{t_{i-1}}^{t_{i}}\left(1+\frac{1}{\varepsilon} e^{-\frac{\alpha t}{\varepsilon}}\right) d t+C \sum_{i=N / 2+1}^{N} h^{(2)} \int_{t_{i-1}}^{t_{i}}\left(1+\frac{1}{\varepsilon} e^{-\frac{\alpha t}{\varepsilon}}\right) d t, \\
\leq C h^{(1)} \int_{0}^{T}\left(1+\frac{1}{\varepsilon} e^{-\frac{\alpha t}{\varepsilon}}\right) d t+C h^{(2)} \int_{0}^{T}\left(1+\frac{1}{\varepsilon} e^{-\frac{\alpha t}{\varepsilon}}\right) d t \\
\leq C\left(h^{(1)}+h^{(2)}\right) \leq C N^{-1} \ln N .
\end{gathered}
$$

Lemma 4.3. For the solution of (4.1)-(4.3), the following estimates hold

$$
\begin{gathered}
\left|\mu^{N}\right| \leq C|r|, \\
\left|z_{0}^{N}\right| \leq|r|+\|c\|_{\infty} T B_{N}\left(\left|\mu^{N}\right| M_{1}+\|R\|_{\infty}\right), \\
\left|z_{i}^{N}\right| \leq\left|z_{0}^{N}\right|+\alpha^{-1}\left(M_{1}\left|\mu^{N}\right|+\|R\|_{\infty}\right), 1 \leq i \leq N-1,
\end{gathered}
$$


where

$$
\begin{aligned}
B_{N} & =\sum_{\ell=1}^{N} \frac{h_{\ell}}{\varepsilon+a_{\ell} h_{\ell}} Q_{N-\ell}, \\
Q_{N-\ell} & = \begin{cases}1, & \text { for } \ell=N, \\
\prod_{s=\ell+1}^{N} \frac{\varepsilon}{\varepsilon+a_{s} h_{s}}, & \text { for } 1 \leq \ell \leq N-1 .\end{cases}
\end{aligned}
$$

Proof. The equation (4.1) can be rewritten as

$$
\varepsilon z_{\bar{t}, i}^{N}+a_{i} z_{i}^{N}=b_{i} \mu^{N}+R_{i}, \quad 1 \leq i \leq N-1,
$$

with

$$
\begin{gathered}
a_{i}=\frac{\partial f}{\partial u}\left(t_{i}, u_{i}+\gamma z_{i}^{N}, \lambda+\gamma \mu^{N}\right), \\
b_{i}=-\frac{\partial f}{\partial \lambda}\left(t_{i}, u_{i}+\gamma z_{i}^{N}, \lambda+\gamma \mu^{N}\right), 0<\gamma<1 .
\end{gathered}
$$

From (4.17) we have

$$
z_{i}^{N}=\frac{\varepsilon}{\varepsilon+a_{i} h_{i}} z_{i-1}^{N}+\mu^{N} \frac{h_{i} b_{i}}{\varepsilon+a_{i} h_{i}}+\frac{h_{i} R_{i}}{\varepsilon+a_{i} h_{i}} .
$$

Solving the first-order difference equation with respect to $z_{i}^{N}$ by using (4.5) and setting the boundary condition (4.3), we get

$$
z_{i}^{N}=-\mu^{N} \sum_{k=i+1}^{N} \frac{h_{k} b_{k}}{\varepsilon+a_{k} h_{k}} Q_{k-i}^{-1}-\sum_{k=i+1}^{N} \frac{h_{k} R_{k}}{\varepsilon+a_{k} h_{k}} Q_{k-i}^{-1} .
$$

Taking into consideration in (4.19) the integral boundary condition (4.2), we have

$$
\begin{gathered}
\mu^{N}=\frac{r}{\sum_{k=1}^{N} \frac{h_{k} b_{k}}{\varepsilon+a_{k} h_{k}} Q_{k}^{-1}+\sum_{k=1}^{N} h_{k} c_{k} \sum_{s=k+1}^{N} \frac{h_{s} b_{s}}{\varepsilon+a_{s} h_{s}} Q_{s-k}^{-1}} \\
+\frac{\sum_{k=1}^{N} \frac{h_{k} R_{k}}{\varepsilon+a_{k} h_{k}} Q_{k}^{-1}+\sum_{k=1}^{N} h_{k} c_{k} \sum_{s=k+1}^{N} \frac{h_{s} R_{s}}{\varepsilon+a_{s} h_{s}} Q_{s-k}^{-1}}{\sum_{k=1}^{N} \frac{h_{k} b_{k}}{\varepsilon+a_{k} h_{k}} Q_{k}^{-1}+\sum_{k=1}^{N} h_{k} c_{k} \sum_{s=k+1}^{N} \frac{h_{s} b_{s}}{\varepsilon+a_{s} h_{s}} Q_{s-k}^{-1}} .
\end{gathered}
$$

Now, we estimate separately the terms on the right-hand side of equality (4.20). For the first term, we have

$$
\begin{gathered}
\left|\frac{r}{\sum_{k=1}^{N} \frac{h_{k} b_{k}}{\varepsilon+a_{k} h_{k}} Q_{k}^{-1}+\sum_{k=1}^{N} h_{k} c_{k} \sum_{s=k+1}^{N} \frac{h_{s} b_{s}}{\varepsilon+a_{s} h_{s}} Q_{s-k}^{-1}}\right| \\
\leq \frac{|r|}{m_{1} \sum_{k=1}^{N} \frac{h_{k}}{\varepsilon+a_{k} h_{k}} Q_{k}^{-1}} \leq \frac{a^{*}|r|}{m_{1} \rho_{*} \sum_{k=1}^{N}\left(1+\rho_{*}\right)^{k-1}}=\frac{a^{*}|r|}{m_{1}\left[\left(1+\rho_{*}\right)^{N}-1\right]},
\end{gathered}
$$


here $\rho_{k}=a_{k} h_{k} / \varepsilon$ and $\rho_{*}=\min \rho_{k}$. Therefore, it is not hard to see that

$$
\left|\frac{r}{\sum_{k=1}^{N} \frac{h_{k} b_{k}}{\varepsilon+a_{k} h_{k}} Q_{k}^{-1}+\sum_{k=1}^{N} h_{k} c_{k} \sum_{s=k+1}^{N} \frac{h_{s} b_{s}}{\varepsilon+a_{s} h_{s}} Q_{s-k}^{-1}}\right| \leq C|r|
$$

Next, evidently

$$
\left|\frac{\sum_{k=1}^{N} \frac{h_{k} R_{k}}{\varepsilon+a_{k} h_{k}} Q_{k}^{-1}+\sum_{k=1}^{N} h_{k} c_{k} \sum_{s=k+1}^{N} \frac{h_{s} R_{s}}{\varepsilon+a_{s} h_{s}} Q_{s-k}^{-1}}{\sum_{k=1}^{N} \frac{h_{k} b_{k}}{\varepsilon+a_{k} h_{k}} Q_{k}^{-1}+\sum_{k=1}^{N} h_{k} c_{k} \sum_{s=k+1}^{N} \frac{h_{s} b_{s}}{\varepsilon+a_{s} h_{s}} Q_{s-k}^{-1}}\right| \leq m_{1}^{-1}\|R\|_{\infty} .
$$

After taking into consideration (4.21) and (4.22) in (4.20), we arrive at (4.14).

Now, we need to estimate $z_{0}$. From (4.18), by using (4.4) we have

$$
z_{i}^{N}=z_{0}^{N} Q_{i}+\mu^{N} \sum_{k=1}^{i} \frac{h_{k} b_{k}}{\varepsilon+a_{k} h_{k}} Q_{i-k}+\sum_{k=1}^{i} \frac{h_{k} R_{k}}{\varepsilon+a_{k} h_{k}} Q_{i-k} .
$$

From here, by virtue of (4.2) it follows that

$$
z_{0}^{N}=\frac{r-\sum_{k=1}^{N} h_{k} c_{k}\left(\mu^{N} \sum_{\ell=1}^{k} \frac{h_{\ell} b_{\ell}}{\varepsilon+a_{\ell} h_{\ell}} Q_{k-\ell}+\sum_{\ell=1}^{k} \frac{h_{\ell} R_{\ell}}{\varepsilon+a_{\ell} h_{k}} Q_{k-\ell}\right)}{1+\sum_{k=1}^{N} h_{k} c_{k} Q_{k}} .
$$

Thereby

$$
\begin{aligned}
\left|z_{0}^{N}\right| \leq & |r|+\|c\|_{\infty} T\left\{\left|\mu^{N}\right| \sum_{\ell=1}^{N} \frac{h_{\ell}\left|b_{\ell}\right|}{\varepsilon+a_{\ell} h_{k}} Q_{N-\ell}+\sum_{\ell=1}^{N} \frac{h_{\ell}\left|R_{\ell}\right|}{\varepsilon+a_{\ell} h_{k}} Q_{k-\ell}\right\}, \\
& \leq|r|+\|c\|_{\infty} T\left\{\left(M_{1}\left|\mu^{N}\right|+\|R\|_{\infty}\right\} \sum_{\ell=1}^{N} \frac{h_{\ell}}{\varepsilon+a_{\ell} h_{\ell}} Q_{N-\ell},\right.
\end{aligned}
$$

which implies validity of (4.15).

Finally, an applying the maximum principle for the difference operator $L^{N} z_{i}^{N}:=$ $\varepsilon z_{\bar{t}, i}^{N}+a_{i} z_{i}^{N}, 1 \leq i \leq N$, to Eq. (4.17) immediately leads to (4.16).

Combining the two previous lemmas gives us the following convergence result.

Theorem 4.1. Let $\{u(t), \lambda\}$ and $\left\{u_{i}^{N}, \lambda^{N}\right\}$ be the exact solution and discrete solution on $\bar{\omega}_{N}$ respectively. Then the following estimates hold

$$
\begin{gathered}
\left|\lambda-\lambda^{N}\right| \leq C N^{-1} \ln N, \\
\left\|u-u^{N}\right\|_{\infty, \varpi_{N}} \leq C N^{-1} \ln N .
\end{gathered}
$$

Proof. This follows immediately by combining the previous lemmas. 


\section{AlgORITHM AND NUMERICAL RESUltS}

Here, we consider a test problem to show the applicability and efficiency of the method described in this paper.

a) We solve the nonlinear problem (3.5)-(3.7) using the following quasilinearization technique:

$$
\begin{gathered}
\lambda^{(n)}=\lambda^{(n-1)}-\frac{\left(B-u_{N-1}^{(n-1)}-u_{i-1}^{(n)}\right) \rho_{N}^{-1}+f\left(T, B, \lambda^{(n-1)}\right)}{\partial f / \partial \lambda\left(T, B, \lambda^{(n)}\right)}, \\
u_{0}^{(n)}=A-c_{N} h_{N} B-\sum_{i=1}^{N-1} h_{i} b_{i} u_{i}^{(n-1)}, \\
u_{i}^{(n)}=u_{i}^{(n-1)}-\frac{\left(u_{i}^{(n-1)}-u_{i-1}^{(n)}\right) \rho_{i}^{-1}+f\left(t_{i}, u_{i}^{(n-1)}, \lambda^{(n)}\right)}{\partial f / \partial u\left(t_{i}, u_{i}^{(n-1)}, \lambda^{(n)}\right)+\rho_{i}^{-1}}, n=1,2, \ldots
\end{gathered}
$$

where, $\rho_{i}=h_{i} / \varepsilon ; \lambda^{(0)}$ and $u_{i}^{(0)}(1 \leq i \leq N-1)$ are the initial iterations given.

b) Consider the test problem:

$$
\begin{gathered}
\varepsilon u^{\prime}+2 u-e^{-u}+t^{2}+\lambda+\tanh (\lambda+t)=0,0<t<1, \\
u(0)+\frac{1}{4} \int_{0}^{1} e^{-s} u(s) d s=1, \\
u(1)=0 .
\end{gathered}
$$

The exact solution of our test problem is not available. Therefore we use the double mesh principle to estimate the errors and to compute the experimental rates of convergence. The error estimates obtained in this way are denoted by

$$
e_{u}^{\varepsilon, N}=\max _{\omega_{N}}\left|u^{\varepsilon, N}-\tilde{u}^{\varepsilon, 2 N}\right|, e_{\lambda}^{\varepsilon, N}=\left|\lambda^{\varepsilon, N}-\tilde{\lambda}^{\varepsilon, 2 N}\right|,
$$

where $\left\{\tilde{u}^{\varepsilon, 2 N}, \tilde{\lambda}^{\varepsilon, 2 N}\right\}$ is the approximate solution on the mesh

$$
\widetilde{\omega}_{2 N}=\left\{t_{i / 2}: i=0,1, \ldots, 2 N\right\}
$$

with $t_{i+1 / 2}=\left(t_{i}+t_{i+1}\right) / 2$ for $i=0,1, \ldots, N-1$. The corresponding rates of convergence are calculated by

for $u$, and

$$
p_{u}^{\varepsilon, N}=\ln \left(e_{u}^{\varepsilon, N} / e_{u}^{\varepsilon, 2 N}\right) / \ln 2
$$

$$
p_{\lambda}^{\varepsilon, N}=\ln \left(e_{\lambda}^{\varepsilon, N} / e_{\lambda}^{\varepsilon, 2 N}\right) / \ln 2
$$

for $\lambda$. The $\varepsilon$-uniform errors $p_{u}^{N}, p_{\lambda}^{N}$ are estimated from

$$
e_{u}^{N}=\max _{\varepsilon} e_{u}^{\varepsilon, N}, e_{\lambda}^{N}=\max _{\varepsilon} e_{\lambda}^{\varepsilon, N} .
$$


The corresponding $\varepsilon$-uniform convergence rates are

$$
p_{u}^{N}=\ln \left(e_{u}^{N} / e_{u}^{2 N}\right) / \ln 2, p_{\lambda}^{N}=\ln \left(e_{\lambda}^{N} / e_{\lambda}^{2 N}\right) / \ln 2 .
$$

In the computations in this section we take $\alpha=2$. The initial guess in the iteration process is taken as $u_{i}^{(0)}=1-t_{i}^{2}, \lambda^{(0)}=-0.4$ and the stopping criterion is

$$
\max _{i}\left|u_{i}^{(n)}-u_{i}^{(n-1)}\right| \leq 10^{-5},\left|\lambda^{(n)}-\lambda^{(n-1)}\right| \leq 10^{-5} .
$$

The values of $\varepsilon$ and $N$ for which we solve the test problem are $\varepsilon=2^{-i}, i=2,4, \ldots, 16$; $N=64,128,256,512,1024$. Some results of numerical experiment are displayed in Tables 1 and 2. The numerical results are the clear illustration of the error estimates.

TABLE 1. Errors $e_{u}^{\varepsilon, N}$ computed $\varepsilon$-uniform errors $e_{u}^{N}$ and convergence rates $p_{u}^{\varepsilon, N}$ on $\omega_{N}$.

\begin{tabular}{clllll}
\hline$\varepsilon$ & $N=64$ & $N=128$ & $N=256$ & $N=512$ & $N=1024$ \\
\hline $2^{-2}$ & 0.00047526 & 0.00025293 & 0.00133677 & 0.00069196 & 0.00035325 \\
& 0.91 & 0.92 & 0.95 & 0.97 & \\
$2^{-4}$ & 0.00477384 & 0.00254057 & 0.00133344 & 0.00069070 & 0.00035261 \\
& 0.91 & 0.93 & 0.95 & 0.97 & \\
$2^{-6}$ & 0.00357052 & 0.00203655 & 0.00115359 & 0.00064445 & 0.00035261 \\
& 0.81 & 0.82 & 0.84 & 0.87 & \\
$2^{-8}$ & 0.00354583 & 0.00203655 & 0.00115359 & 0.00064445 & 0.00035261 \\
& 0.80 & 0.82 & 0.84 & 0.87 & \\
$2^{-10}$ & 0.00349702 & 0.00203655 & 0.00115359 & 0.00064445 & 0.00035261 \\
& 0.78 & 0.82 & 0.84 & 0.87 & \\
$2^{-12}$ & 0.00349702 & 0.00203655 & 0.00115359 & 0.00064445 & 0.00035261 \\
& 0.78 & 0.82 & 0.84 & 0.87 & \\
$2^{-14}$ & 0.00349702 & 0.00203655 & 0.00115359 & 0.00064445 & 0.00035261 \\
& 0.78 & 0.82 & 0.84 & 0.87 & \\
$2^{-16}$ & 0.00349702 & 0.00203655 & 0.00115359 & 0.00064445 & 0.00035261 \\
& 0.78 & 0.82 & 0.84 & 0.87 & \\
$e_{u}^{\varepsilon, N}$ & 0.00349702 & 0.00203655 & 0.00133677 & 0.00069196 & 0.00035261 \\
$p_{u}^{\varepsilon, N}$ & 0.78 & 0.82 & 0.84 & 0.97 & \\
\hline
\end{tabular}


TABLE 2. Errors $e_{\lambda}^{\varepsilon, N}$ computed $\varepsilon$-uniform errors $e_{\lambda}^{N}$ and convergence rates $p_{\lambda}^{\varepsilon, N}$ on $\omega_{N}$.

\begin{tabular}{clllll}
\hline$\varepsilon$ & $N=64$ & $N=128$ & $N=256$ & $N=512$ & $N=1024$ \\
\hline $2^{-2}$ & 0.03255711 & 0.01831433 & 0.01002063 & 0.00533283 & 0.00276045 \\
& 0.83 & 0.87 & 0.91 & 0.95 & \\
$2^{-4}$ & 0.03134362 & 0.01838045 & 0.01048388 & 0.00581630 & 0.00313856 \\
& 0.77 & 0.81 & 0.85 & 0.89 & \\
$2^{-6}$ & 0.03134362 & 0.01838045 & 0.01048388 & 0.00581630 & 0.00313856 \\
& 0.77 & 0.81 & 0.85 & 0.89 & \\
$2^{-8}$ & 0.03134362 & 0.01838045 & 0.01048388 & 0.00581630 & 0.00313856 \\
& 0.77 & 0.81 & 0.85 & 0.89 & \\
$2^{-10}$ & 0.03134362 & 0.01838045 & 0.01048388 & 0.00581630 & 0.00313856 \\
& 0.77 & 0.81 & 0.85 & 0.89 & \\
$2^{-12}$ & 0.03134362 & 0.01838045 & 0.01048388 & 0.00581630 & 0.00313856 \\
& 0.77 & 0.81 & 0.85 & 0.89 & \\
$2^{-14}$ & 0.03134362 & 0.01838045 & 0.01048388 & 0.00581630 & 0.00313856 \\
& 0.77 & 0.81 & 0.85 & 0.89 & \\
$2^{-16}$ & 0.03134362 & 0.01838045 & 0.01048388 & 0.00581630 & 0.00313856 \\
& 0.77 & 0.81 & 0.85 & 0.89 & \\
$e_{\lambda}^{\varepsilon, N}$ & 0.03134362 & 0.01838045 & 0.01048388 & 0.00581630 & 0.00313856 \\
$p_{\lambda}^{\varepsilon, N}$ & 0.77 & 0.81 & 0.85 & 0.89 & \\
\hline
\end{tabular}

\section{CONCLUSION}

A parameterized singular perturbation problem with integral boundary condition is considered. The difference scheme is constructed by the method of integral identities with the use of interpolating quadrature rules with the weight and remainder terms in integral form. The numerical method presented here comprises a backward difference operator on a non-uniform mesh for the equation and composite rectangle rule for the integral condition. It is shown that the method displays uniform convergence with respect to the perturbation parameter. Numerical results confirm our theoretical analysis. The main lines for the analysis of the uniform convergence carried out here can be used for the study of more complicated nonlinear singularly perturbed analogous type problems. 


\section{REFERENCES}

[1] G. M. Amiraliyev, I. G. Amiraliyeva, and M. Kudu, "A numerical treatment for singularly perturbed differential equations with integral boundary condition." Appl. Math. Comput., vol. 185, no. 1, pp. 574-582, 2007, doi: 10.1016/j.amc.2006.07.060.

[2] G. M. Amiraliyev and H. Duru, "A note on a parameterized singular perturbation problem," J.Compu. Appl. Math., vol. 182, no. 1, pp. 233-242, 2005, doi: 10.1016/j.cam.2004.11.047.

[3] G. M. Amiraliyev, M. Kudu, and H. Duru, "Uniform difference method for a parameterized singular perturbation problem.” Appl. Math. Comput., vol. 175, no. 1, pp. 89-100, 2006, doi: 10.1016/j.amc.2005.07.068.

[4] N. Borovykh, "Stability in the numerical solution of the heat equation with nonlocal boundary conditions." Appl. Numer. Math., vol. 42, no. 1-3, pp. 17-27, 2002, doi: 10.1016/s01689274(01)00139-8.

[5] M. Cakir and G. M. Amiraliyev, "Numerical solution of a singularly perturbed three-point boundary value problem." Int. J. Comput. Math., vol. 84, no. 10, pp. 1465-1481, 2007, doi: 10.1080/00207160701296462.

[6] J. R. Cannon, "The solution of the heat equation subject to the specification of energy," Quart. Appl. Math., vol. 21, no. 2, pp. 155-160, 1963, doi: 10.1090/qam/160437.

[7] Z. Cen and X. Cai, "A second-order upwind difference scheme for a singularly perturbed problem with integral boundary condition in netural network," Lecture Notes in Computer Science,Springer Berlin Heidelberg, pp. 175-181, 2007, doi: 10.1007/978-3-540-74827-4'22.

[8] M. Chandru, T. Prabha, P. Das, and V. Shanthi, "A numerical method for solving boundary and interior layers dominated parabolic problems with discontinuous convection coefficient and source terms." Differ. Equ. Dyn. Syst., pp. 1-22, 2017, doi: 10.1007/s12591-017-0385-3.

[9] P. Das, "Comparison of a priori and a posteriori meshes for singularly perturbed nonlinear parameterized problems," J. Comput. Appl. Math., vol. 290, pp. 16-25, 2015, doi: 10.1016/j.cam.2015.04.034.

[10] P. Das and S. Natesan, "Numerical solution of a system of singularly perturbed convection diffusion boundary value problems using mesh equidistribution technique." AJMAA, vol. 10, no. 1, pp. 1-17, 2013.

[11] P. Das and S. Natesan, "Richardson extrapolation method for singularly perturbed convectiondiffusion problems on adaptively generated mesh." CMES, vol. 90, no. 6, pp. 463-485, 2013.

[12] P. A. Farrel, A. F. Hegarty, J. J. H. Miller, E. O'Riordan, and G. I. Shishkin, Robust Computational Techniques for Boundary Layers. New York: Chapman and Hall/CRC, 2000.

[13] M. Feckan, "Parametrized singularly perturbed boundary value problems." J. Math. Anal. and Appl., no. 2, pp. 426-435, 1994, doi: 10.1006/jmaa.1994.1436.

[14] J. Henderson, R. Luca, and A. Tudorache, "Positive solutions for a system of second-order differential equations with integral boundary conditions." IJAPM, vol. 6, no. 3, pp. 138-149, 2016, doi: 10.17706/ijapm.2016.6.3.138-149.

[15] N. I. Ionkin, "The solution of a certain boundary value problem of the theory of heat conduction with a nonclassical boundary condition.” Dif. Eqs., vol. 13, no. 2, pp. 294-304, 1977.

[16] T. Jankowski, "Application of the numerical-analitical method to systems of differential equations with a parameter." Ukrain. Math. J., vol. 54, no. 4, pp. 671-683, 2002, doi: 10.1023/a:1021043629726.

[17] S. Kumar and M. Kumar, "A second order uniformly convergent numerical scheme for parameterized singularly perturbed delay differential problems." Numer. Algor., vol. 76, no. 2, pp. 349-360, 2016, doi: 10.1007/s11075-016-0258-9. 
[18] X. Liu and F. A. McRae, "A monotone iterative method for boundary value problems of parametric differential equations." J. Appl. Math. Stoch. Anal., vol. 14, no. 2, pp. 183-187, 2001, doi: 10.1155/s1048953301000132.

[19] J. J. H. Miller, E. O’Riordan, and G. I. Shishkin, Fitted Numerical Methods for Singular Perturbation Problems. Singapore: World Scientific Publishing Co. Pte. Ltd., 2012.

[20] F. Nicoud and T. Schönfeld, "Integral boundary conditions for unsteady biomedical CFD applications." Int. J. Numer. Methods Fluids, vol. 40, no. 3-4, pp. 457-465, 2002, doi: 10.1002/fld.299.

[21] R. E. O'Malley, Singular Perturbation Methods for Ordinary Differential Equations. New York: Springer-Verlag, 1991.

[22] T. Pomentale, "A constructive theorem of existence and uniqueness for the problem $y^{\prime}=$ $f(x, y, \lambda), y(a)=\alpha, y(b)=\beta$." Z. Angew. Math. Mech., vol. 56, no. 8, pp. 387-388, 1976, doi: 10.1002/zamm.19760560806.

[23] A. M. Samoilenko and S. V. Martynyuk, "Justification of the numerical-analytic method of successive approximations for problems with integral boundary conditions." Ukrain. Mat. Zh. SSR, vol. 43, pp. 1231-1239, 1991.

[24] Y. Wang, S. Chen, and X. Wu, "A rational spectral collocation method for solving a class of parameterized singular perturbation problems." J. Comput. Appl. Math., vol. 233, no. 10, pp. 2652 2660, 2010, doi: 10.1016/j.cam.2009.11.011.

[25] F. Xie, J. Wang, W. Zhang, and M. He, "A novel method for a class of parameterized singularly perturbed boundary value problems." J. Comput. Appl. Math., vol. 213, no. 1, pp. 258-267, 2008, doi: 10.1016/j.cam.2007.01.014.

\section{Authors' addresses}

\section{Mustafa Kudu}

Department of Mathematics, Faculty of Arts and Sciences, Erzincan University, 24100, Erzincan, Turkey

E-mail address: muskud28@yahoo.com

\section{Ilhame Amirali}

Department of Mathematics, Faculty of Arts and Sciences, Düzce University, 81620, Düzce, Turkey

E-mail address: ailhame@gmail.com

\section{Gabil M. Amiraliyev}

Department of Mathematics, Faculty of Arts and Sciences, Erzincan University, 24100, Erzincan, Turkey

E-mail address: gabilamirali@yahoo.com 